Research Article

\title{
Encapsulation Based Method for Natural Frequency Identification of Deployable Solar Arrays with Multiple Plates
}

\author{
Chunjuan Zhao $\mathbb{D}^{1,2,3}$ Xiangyu Zhao $\mathbb{D}^{1,},{ }^{1,2,3}$ Shanbo Chen $\mathbb{D}^{\circ},{ }^{3}$ Jisong Yu $\mathbb{D}^{1,},{ }^{1,2,3}$ \\ and Lei Zhang iD $^{3}$ \\ ${ }^{1}$ Changchun Institute of Optics, Fine Mechanics and Physics, Chinese Academy of Sciences, Changchun 130033, China \\ ${ }^{2}$ University of Chinese Academy of Sciences, Beijing 100049, China \\ ${ }^{3}$ Satellite Structure Research Laboratory, Chang Guang Satellite Technology Co., Ltd., Changchun 130033, China
}

Correspondence should be addressed to Lei Zhang; 18686344285@163.com

Received 30 August 2021; Revised 30 October 2021; Accepted 10 November 2021; Published 26 November 2021

Academic Editor: Jiaxi Zhou

Copyright $(2021$ Chunjuan Zhao et al. This is an open access article distributed under the Creative Commons Attribution License, which permits unrestricted use, distribution, and reproduction in any medium, provided the original work is properly cited.

The ground modal test is an important approach to the natural frequency of solar arrays to support the attitude control of spacecraft. However, for the batch production of small satellites, the accuracy and efficiency of traditional ground modal testing methods are limited. This shortcoming restricts the development of satellite constellations. Based on the encapsulation method widely used in the computer field, this paper proposed a natural frequency identification method of deployable solar arrays with multiple plates. This method is of high accuracy and efficiency that meets the demand of attitude control and makes sense to accelerate the batch production of small satellites. First, a suspended modal test system with gravity compensation function is designed. Second, the mathematical model of the test system is established. Abstracting parts of the parameters of the test object into an encapsulated entity, the mathematical model is simplified by equivalent variables. Thus, the direct mapping relationship between the ground test result and the true natural frequency is proposed. Finally, to verify the identification accuracy, finite element analysis (FEA) and the ground modal test of a two-folder solar array simulant are carried out. The results show that the relative error of the first-order natural frequency after correction and the theoretical value is less than $3 \%$. Meanwhile, the identification accuracy of the ground modal test is improved by more than $50 \%$. This method improves the availability of ground test results and reduces the calculation amount, so that it is convenient for engineering applications.

\section{Introduction}

The satellite ground test is an effective means to comprehensively test the functions and performance of the satellite and its subsystems. Moreover, it provides an important basis for subsequent analysis and optimization. Therefore, the ground test is an essential step in the process of satellite manufacture. With the rapid development of satellite constellations, such as Starlink, the manufacture of small satellites has officially entered the batch mode. Test efficiency has gradually become one of the key factors restricting the speed of satellite development [1-3]. The previous test methods are not suitable because currently small satellites are manufactured with diverse models, large quantity, and short production cycles. Thus, test methods with complex processes should be replaced. Furthermore, for attitude control of spacecraft, the disturbances generated by flexible structures such as solar arrays and large antennas will affect the system's dynamics [4]. To suppress the disturbances, the active control parameters need to be designed according to the natural frequency of the flexible structure $[5,6]$. The ground modal test is an important approach of the natural frequency of flexible structures. However, due to the environmental differences between ground and space, the modal parameters obtained from ground tests often cannot accurately reflect the natural frequency of spacecraft $[7,8]$. To obtain usable modal parameters of deployable solar arrays, several mainstream solutions have been developed. Nevertheless, these methods do not meet the requirement of batch small satellites due to their limitations and low efficiency. 
The first type of method is to optimize the test system and reduce the systematic error caused by ground test conditions. Woodard used the combination of various springs and structures to form a suspension device, which was named "ZSRM" and had approximately zero stiffness. However, the disadvantage of this device is that the adjustable stroke is short and the motion range is limited [9]. Ashory proposed a SMURF method based on direct substructure technology. This method counteracted the load effect caused by the mass of sensors and corrected the influence of the spring suspension on the test structure. However, this method has defects in the application of noise data [10]. Hunady and Hagara used the high-speed camera for modal testing [11], and Kumar et al. developed a 3D digital image system based on FPGA [12]. Both of them used the $3 \mathrm{D}$ data image to determine the modal parameters of the mechanical system. Thus, the noncontact testing of light and flexible structures was achieved, and the introduction of the added sensor mass was avoided. Nevertheless, the test accuracy of the 3D image system is limited by the size of test objects. The accuracy decreases with the increase of the image range. Zhang et al. carried out the modal identification test of membrane structure through the low-vacuum test device. Besides, the influences on modal identification results of observation points and the amplitude of vibration exciter were discussed through simulation analysis [13]. To recreate the space environment during the ground test as much as possible, many research institutions and scholars have proposed different test methods. But it is always difficult to eliminate all systematic errors caused by the ground environment and the composition of the test system at the same time.

Generally, the ground modal test results cannot be used directly. Therefore, it is necessary to combine the second type of method, that is, the correction of the systematic errors. Usually, a finite element model for model updating or a theoretical model for error estimation is established $[14,15]$. The accurate parameters in models are obtained by a series of tests. Wickramasinghe et al. carried out the testing modal analysis based on the multi-input multi-output (MIMO) method on the model of CASSIOPE. They used the finite element method (FEM) to obtain the dynamics information, while the model updating accuracy was only within $10 \%$ [16]. Luo et al. performed FEM model matching of the satellite sailboard based on sensitivity analysis. The low-order frequency errors in the simulation model were less than 5\%, and the high-order errors were less than $10 \%$ [17]. For the solar array of multiple plates, the parameter number is large, while the accuracy of the FEM model is insufficient. In addition, the on-orbit testing is carried out and is helpful to the design of solar arrays of the same type. Xie et al. achieved on-orbit identification of the natural frequency of solar arrays by using the spacecraft's attitude maneuver data and acceleration signal [18]. Tang et al. proposed a modal identification method based on stochastic resonance. This method took the reaction flywheel as the exciter and collected and analyzed the vibration signals through sensors and controllers of the active control system [19]. However, the on-orbit method is far behind the manufacture of spacecraft, thus could only work on future products. Moreover, Zang et al. and Wu et al. both used the Rayleigh method to study the influence of the ground test system on the modal testing structure [20, 21]. However, these theoretical models only provided a rough estimation for parts of the systematic errors. The accuracy is limited when they are applied to multiple-plate structures directly.

In summary, the first type of method only helps reduce parts of the testing error in specific cases, and it is lack of universality. The second type of method usually requires a premastery of the objects' parameters, while the parameter accuracy is limited. Besides, it belongs to the post-analysis method of test data. The lag degree varies with different means, but the test efficiency is inevitably reduced.

Consequently, most of the traditional methods have disadvantages of complex systems, complex model updating process, time-consuming, large data lag, and poor flexibility. They are typically low-efficiency test systems. Therefore, to meet the needs of attitude control and the batch production of small satellites, it is significant to research on efficient ground modal testing methods of solar arrays. To ensure the usability of natural frequency data from ground tests, error correction is an essential process. However, it will improve the identification efficiency if the need for the information of measured objects can be reduced during the correction. Thus, fewer data are used for the identification of natural frequencies. The encapsulation method is widely used in the computer field, which means hiding the attributes and internal details of the object. It leaves only part of the external interface to allow contact with the outside. First, abstract parts of the structural parameters of solar arrays using the encapsulation method and make them into an inseparable and independent entity. The interaction is performed only on other parameters and this entity. Subsequently, the encapsulated entity is used as a medium to establish the direct mapping relationship between the modal test results and the true natural frequency. With this method, the high-accuracy identification of natural frequencies is achieved on the premise of limited system information.

In this paper, a high-efficiency natural frequency identification method is proposed for one kind of current mainstream solar array, deployable solar arrays with multiple plates. The method has two advantages over traditional methods. First, parts of structural parameters of solar arrays are hidden based on the encapsulation method to avoid the complex processes of parameter testing and model updating. Second, the error correction is achieved through the mathematical model with low calculation. Thus, the proposed identification method not only improves the test efficiency but also ensures the accuracy of identification.

\section{Test System and Mathematical Model}

Accurate measurement of modal information is important for dynamic modeling and structural optimization. For onorbit satellites, inaccurate estimation of the natural frequency for flexible structures may lead to the decrease of attitude control accuracy and even the instability of the satellite. Especially, the first-order natural frequency that 
takes high energy will impact on satellite attitude greatly in the stabilization phase and it deserves attention. The modal test accuracy is determined by many factors, such as equipment, software, personnel, and environment, and so on. Therefore, the ground test result cannot be applied to spacecraft dynamics analysis directly.

2.1. Suspended Modal Test System. For high-power spacecraft, the deployable solar array with multiple plates is one kind of mainstream configuration. It is formed by modularized substructures in sequence. The substructure has features of large amount, lightweight, and low frequency. To obtain the correct modal parameters of solar arrays, the ground modal test should recreate the space environment of the on-orbit spacecraft as much as possible. The zero-gravity characteristic is an obvious difference distinguishing the space environment from the ground environment. Therefore, the primary problem of ground modal tests is to achieve gravity compensation of the test object and satisfy free boundary conditions as much as possible. The common methods are the air floating method and the suspension method, as well as the aerodynamic suspension method, which is the combination of the above two methods. For flexible solar arrays, some scholars used the inverted deployment method. Due to the influence of gravity, the hinge stiffness will be increased by the inverted deployment method. The free boundary conditions cannot be fully simulated by the air flotation method, and the added mass of the aerodynamic suspension method is large. Besides, the pneumatic components lag behind solar arrays due to the inertial force. Considering the structural characteristics of deployable solar arrays with multiple plates, a suspension rope is used to compensate for the gravity of the solar array. The spring is set on the upper of each rope to simulate the free boundary condition. Thus, the test system has a small added mass and is easy to expand.

The modal test system adopts the MIMO hammering method that uses the force hammer as the exciter. Lowfrequency acceleration sensors are pasted on the surface of the solar array substructure. The system composition is shown in Figure 1, mainly including large truss, fixed equipment, spring, force sensor, height adjusting module, rope, data acquisition and signal processing system (DASP), etc. The force sensor is connected to the rope with the height adjusting module to achieve the accurate gravity compensation of the substructure. The upper end of the rope is connected fixedly to avoid the uncertainty caused by the asynchronous motion of the gravity compensation component, thereby be beneficial to establish the mathematical model of the suspension system.

2.2. Establishment of the Mathematical Model. The error source of the suspended test system is analyzed, and the mathematical model of the first-order natural frequency is established containing error factors of the ground test. Then the model is rationally simplified to achieve the encapsulation of solar array parameters.

In the suspended modal test system, the systematic error caused by the ground test conditions mainly includes the following factors:

(1) The error caused by the suspension spring and the rope when simulating free boundary conditions. Therein, the spring stiffness and the rope stiffness will affect the horizontal and the vertical frequency characteristics of solar arrays. In addition, the rope swing will produce the horizontal component force, which affects the horizontal frequency characteristics.

(2) The error caused by the added mass of force sensors, accelerometers, height adjusting modules, and other attachments. It will affect the natural frequency in the vertical and the horizontal directions.

Therefore, the above error factors are selected as research objects and the mathematical model of the suspended modal test system is established. Then the formulas of first-order natural frequencies in the horizontal and the vertical directions can be acquired.

\subsubsection{Mathematical Model in the Horizontal Direction.} In the suspended modal test system, the suspension rope will produce a pendulum effect. The theoretical model is shown in Figure 2.

The horizontal component $k_{s x}$ of the spring stiffness $k$ satisfies:

$$
k_{s x}=\frac{\Delta T \sin \theta}{x}=\frac{\Delta T}{x / \sin \theta}=\frac{\Delta T}{\Delta L}=k .
$$

The relationship between the swing angle and the restoring force of the pendulum satisfies:

$$
F=G \tan \theta=\frac{m g}{L} x,
$$

where $L$ is the rope length. The function of the restoring force of the pendulum is equivalent to adding a serial spring $m g / L$ with the spring $k$.

The environment error of the first-order natural frequency in the horizontal direction is mainly caused by the rope swing, the added stiffness, and the added mass. The theoretical model of the horizontal vibration of the solar array is shown in Figure 3.

The kinetic equation is given as

$$
M \ddot{x}+C_{x} \dot{x}+K_{x} x=F_{x} .
$$

In the small damping system, the damping mainly affects the amplitude, while has little effect on the natural frequency. So the damping is ignored. In equation (3), there is 


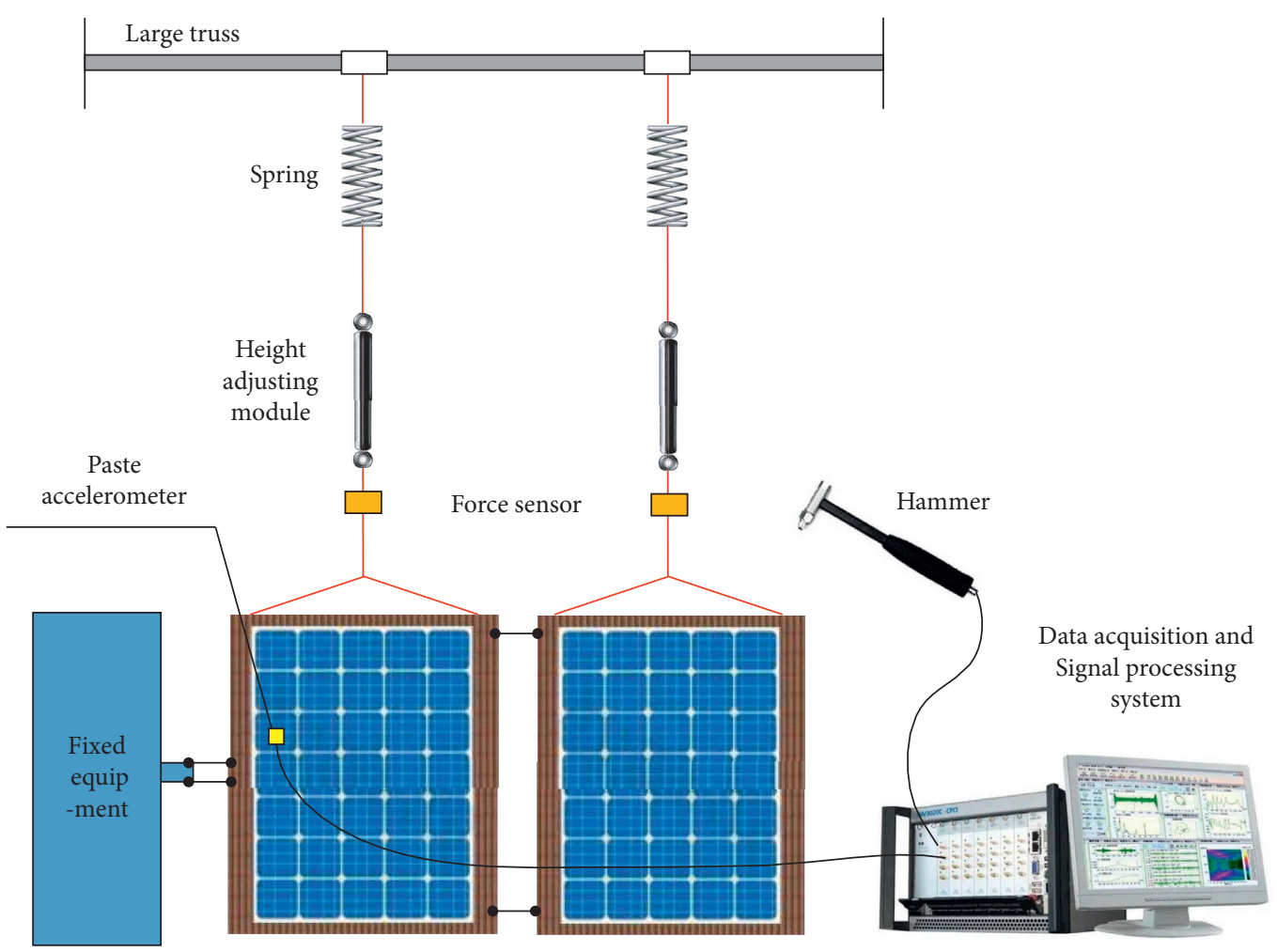

Figure 1: The suspended modal test system of solar arrays.

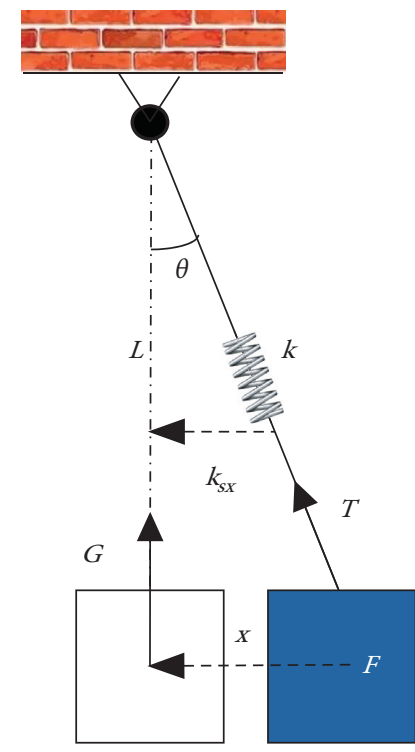

Figure 2: The theoretical model of rope pendulum. 


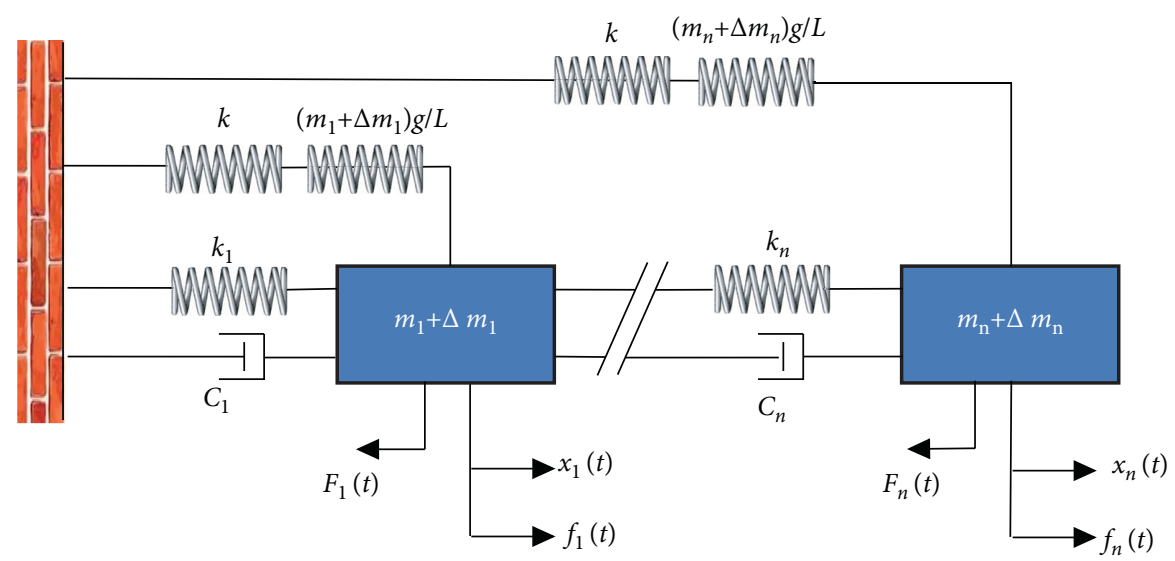

Figure 3: The theoretical model of the horizontal vibration of deployable solar arrays with multiple plates.

$$
\begin{aligned}
& M=\left[\begin{array}{ccccc}
m_{1}+\Delta m_{1} & & & & \\
& \ddots & & & \\
& & m_{i}+\Delta m_{i} & & \\
& & & \ddots & \\
& & & & m_{n}+\Delta m_{n}
\end{array}\right] \text {, }
\end{aligned}
$$

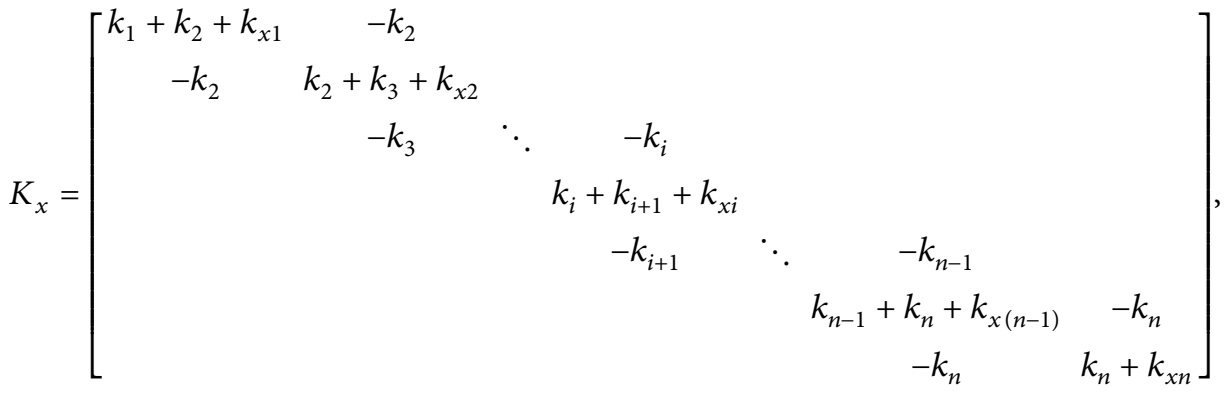

$$
\begin{aligned}
& \omega_{x}=f\left(m_{i}, \Delta m_{i}, k_{\mathrm{eq} x}, k, L\right) .
\end{aligned}
$$

in which, $m_{i}$ is the substructure mass; $\Delta m_{i}$ is the added mass caused by attachments; $k_{i}$ is the substructure stiffness in the horizontal direction; $k_{x i}=1 /\left((1 / k)+L /\left(m_{i}+\Delta m_{i}\right) g\right)$, which is the added stiffness in the horizontal direction; $k$ is the suspending stiffness, determined by the spring stiffness and the rope stiffness collectively.

The natural circular frequency $\omega_{x}$ of the system satisfies:

$$
\left|K_{x}-\omega_{x}^{2} M\right|=0 .
$$

Therefore, the first-order natural circular frequency in the horizontal direction measured by the suspended modal test system is the function of the following variables:

$$
\omega_{x}=f\left(m_{i}, \Delta m_{i}, k_{i}, k, L\right) .
$$

For deployable solar arrays with multiple plates, the difference of the substructures' stiffness is mainly caused by the discreteness of materials and the manufacturing process. Let $k_{i}=k_{\text {eq } x}(i=1,2, \ldots, n)$ (where $k_{\text {eq } x}$ is the equivalent stiffness of the substructure in the horizontal direction), the simplified mathematical model of the natural circular frequency in the horizontal direction under the testing state can be obtained as
2.2.2. Mathematical Model in the Vertical Direction. The systematic error of the first-order natural frequency in the vertical direction is mainly caused by the suspending stiffness and the added mass. The theoretical model of the vertical vibration of deployable solar arrays with multiple plates is shown in Figure 4.

The kinetic equation is given as

$$
M \ddot{y}+C_{y} \dot{y}+K_{y} y=F_{y} .
$$

The effect on the natural frequency of the small damping is ignored. The kinetic equation is similar to the equation in Section 2.2.1. Replace the $k_{x}$ in the stiffness matrix $K_{x}$ with $k_{y}=k$, and the stiffness matrix $K_{y}$ is obtained. Then, the natural circular frequency $\omega_{y}$ of the system satisfies:

$$
\left|K_{y}-\omega_{y}^{2} M\right|=0
$$

Therefore, the first-order natural circular frequency in the vertical direction measured by the suspended modal test system is the function of the following variables: 


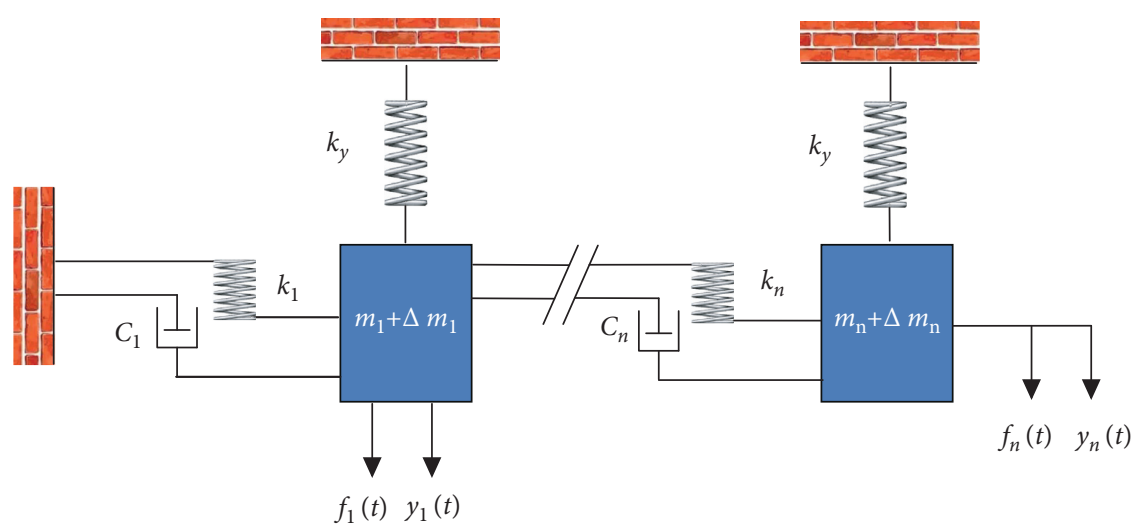

FIgURE 4: The theoretical model of the vertical vibration of deployable solar arrays with multiple plates.

$$
\omega_{y}=f\left(m_{j}, \Delta m_{j}, k_{j}, k\right) .
$$

Let $k_{j}=k_{\text {eq } y}(i=1,2, \ldots, n)$ (where $k_{\text {eq } y}$ is the equivalent stiffness of the substructure in the vertical direction), and the simplified mathematical model of the natural circular frequency in the vertical direction under the testing state can be obtained as

$$
\omega_{y}=f\left(m_{j}, \Delta m_{j}, k_{\text {eq } y}, k\right) .
$$

2.3. Strategy of Encapsulation and Mapping. The kinematic equation of the on-orbit solar array is given as

$$
M \ddot{x}+C \dot{x}+K x=F .
$$

The true stiffness matrix $K$ is encapsulated as the equivalent stiffness matrix $K_{\text {eq }}=\operatorname{diag}\left(k_{\text {eq }}, \ldots, k_{\text {eq }}\right)$. Since the substructure mass can be measured, the true value of first-order natural frequency can be simplified as a function of the equivalent stiffness $K_{\mathrm{eq}}$ of the substructure, i.e.:

$$
f=\frac{1}{2 \pi} \omega=\frac{1}{2 \pi} f\left(k_{\mathrm{eq}}\right) \text {. }
$$

In the same way, the substructure stiffness $k_{i}$ and $k_{j}$ are encapsulated according to the simplified model of the test system obtained in Sections 2.2.1 and 2.2.2. Because the substructure parameters $m, \Delta m$ can be measured, and the suspension stiffness $k$ and the suspension rope length $L$ can be designed, it can be inferred that the ground test value of the natural frequency is also a function of the equivalent stiffness of the substructure.

Under the ground test conditions, the first-order natural frequency of the solar array measured by the suspended modal test system in the horizontal direction is given as

$$
f_{x}=\frac{1}{2 \pi} f_{x}\left(k_{\mathrm{eq} x}\right) \text {. }
$$

The first-order natural frequency in the vertical direction is given as

$$
f_{y}=\frac{1}{2 \pi} f_{y}\left(k_{\text {eq } y}\right)
$$

Stiffness characteristics of the substructure are hidden. The true stiffness matrix and the test stiffness matrix of solar arrays are expressed with the equivalent stiffness. In this way, the encapsulated mathematical model can be used as a medium to map the ground modal test result with the true natural frequency of solar arrays, as shown in Figure 5. The detailed method is as follows: First, substitute the test result into equations (14) and (15), respectively, to obtain the equivalent stiffness of the substructure. Then substitute the equivalent stiffness into equations (13) to compensate for the systematic error of the first-order natural frequency caused by the ground test conditions. Thus, the natural frequency of the solar array is obtained.

The process comparison with the traditional correction method based on model updating is shown in Figure 6. It is clear that the encapsulation-based identification method proposed in this paper shortens the error correction process. The time and complexity of natural frequency identification will be reduced, and this makes sense to the batch production of small satellites.

\section{Simulation Verification}

In current mainstream types of solar arrays, the ones of high utilization should be analyzed as cases to verify the universality of the identification method proposed in this paper. To meet the above requirements, the configurations are selected as two folds and three folds, the substrate materials are selected as printed circuit board (PCB) and composite material, and the size magnitudes are selected as $0.5 \mathrm{~m}$ and $1 \mathrm{~m}$. The FEM models of deployable solar arrays with multiple plates are established with different fold numbers $(n=2,3)$. Then choose parts of parameters affecting the independent variables $k_{i}, k_{j}$, and $m_{i}\left(m_{j}\right)$ in the mathematical model and set a certain discreteness artificially, as shown in Table 1 . That is, make $k_{i}, k_{j}$, and $m$ in each example not completely equal. The modal analysis results in the ideal state are taken as theoretical values. The first-order natural frequencies measured by the suspended modal test system can be analyzed through simulation. Subsequently, compare the true natural frequencies with the natural frequencies identified according to the test results. 


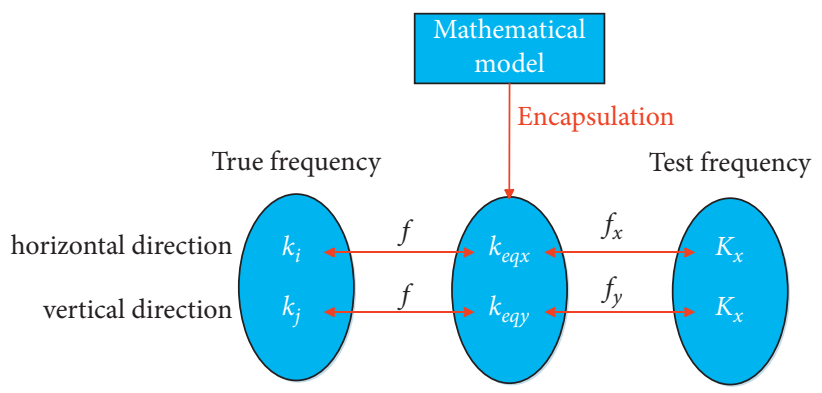

Figure 5: Mapping strategy.

TABLE 1: Discretization of structural parameters.

\begin{tabular}{lccc}
\hline Structural parameter & Single plate & & Hinge \\
Related variable & Density & Elastic modulus & Stiffness \\
\hline
\end{tabular}

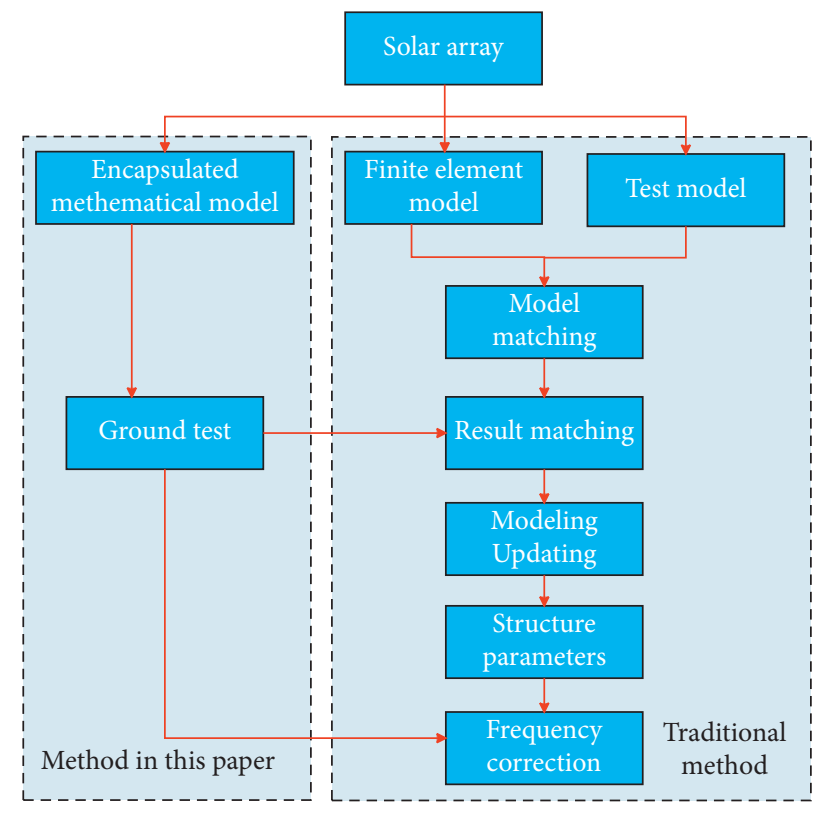

Figure 6: Process comparison.

3.1. Twofold Solar Array of PCB Plates. There is a deployable solar array of two folds applicable to a cubic satellite. Its substrates adopt PCB plates. For the plate, the single size is about $386 \mathrm{~mm} \times 440 \mathrm{~mm}$ that weights about $960 \mathrm{~g} \sim 973 \mathrm{~g}$, and the deployable size is about $790 \mathrm{~mm}$. The mass of a single hinge is about $12 \mathrm{~g}$. Parts of the parameters are discretized, and the first three-order modes are obtained according to FEM, as shown in Figure 7. Therein, Mode 1 shows the firstorder natural frequency in the horizontal direction, and Mode 3 shows the first-order natural frequency in the vertical direction.

The FEM model of the ground modal test system is established for the twofold solar array of PCB plates, as shown in Figure 8. In the simulation, the gravity load is applied to the test system. A force load equal to and opposite to gravity is applied on the node below the spring element.
Set the static analysis results under gravity as the preload in modal analysis.

Test values of the first-order natural frequencies in the horizontal and the vertical directions are obtained under different test conditions. Using the test values, the true natural frequencies of the solar array are identified and compared with the theoretical values, as shown in Tables 2 and 3. For analysis, the suspension stiffness, determined by the stiffness of the spring and the rope, is about multiples of $0.1 \mathrm{~N} \cdot \mathrm{mm}^{-1}$, and the rope length is about times of the plate length.

In Tables 2 and 3, it should be noted that the relative test error approaches 0 , not when the suspension rope length and the suspension stiffness approach infinity, but when they take certain intermediate values. This phenomenon cannot be understood as that the current test system is error free. It 


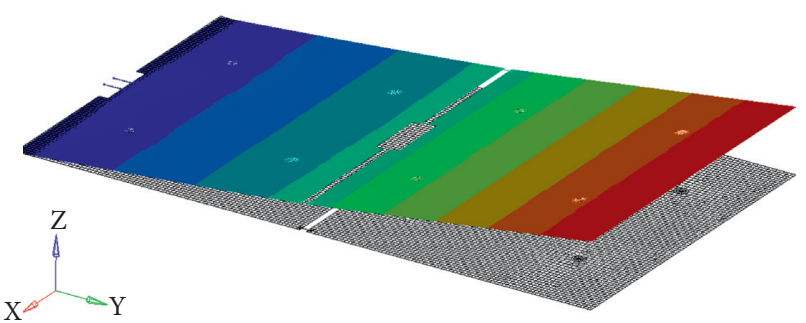

(a)

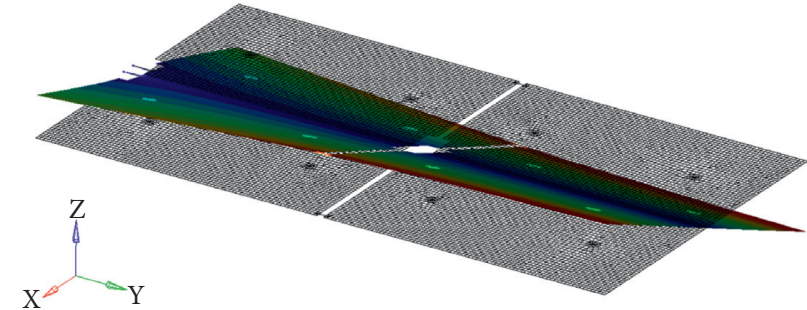

(b)

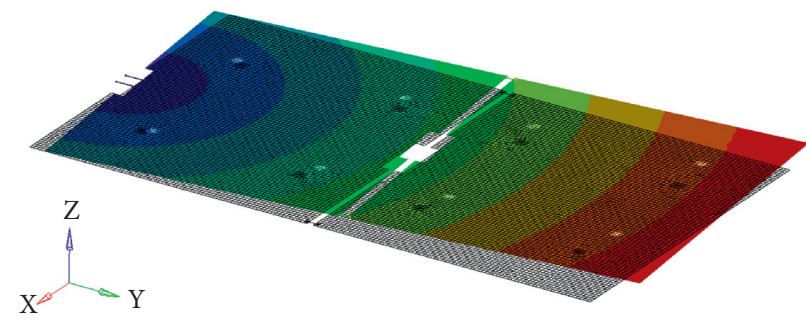

(c)

FIGURE 7: First three-order modes of the twofold solar array of PCB plates: (a) mode 1: freq. $=0.7377 \mathrm{~Hz}$; (b) mode 2: freq. = $3.2057 \mathrm{~Hz}$; (c) mode 3: freq. $=3.5942 \mathrm{~Hz}$.

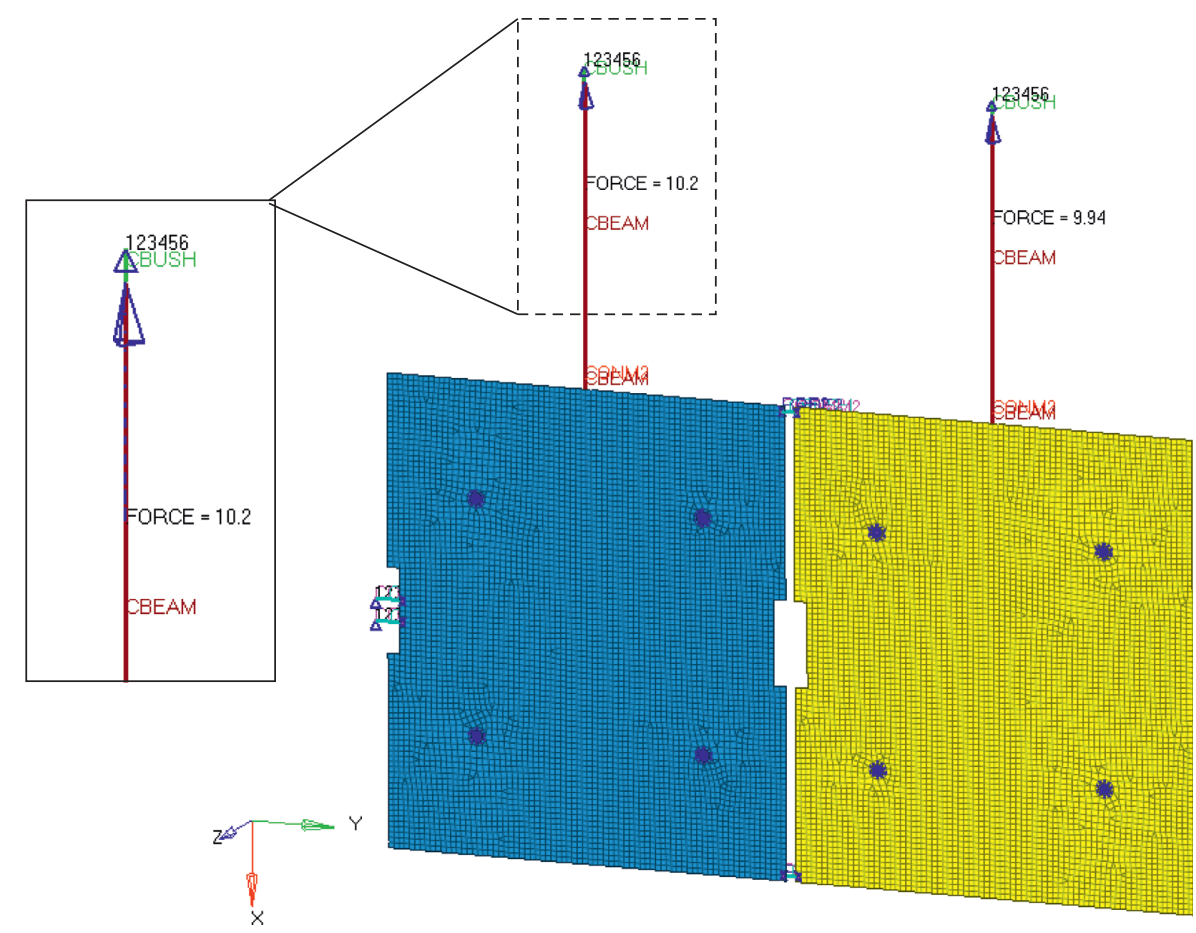

Figure 8: Finite element model of the test system.

TABLE 2: Natural frequencies of the twofold solar array in the horizontal direction.

First-order natural frequency in the horizontal direction

Rope length $L(\mathrm{~m})$ Suspension stiffness $k_{x}\left(\mathrm{~N} \cdot \mathrm{mm}^{-1}\right)$ Test value Relative error of Corrected value of mathematical Corrected relative

\begin{tabular}{lcccccc} 
& & $(\mathrm{Hz})$ & test $(\%)$ & \multicolumn{1}{c}{ model $(\mathrm{Hz})$} & -0.49 \\
0.5 & 0.098 & 0.9545 & 29.39 & 0.7341 & 0.19 \\
1.0 & 0.097 & 0.8558 & 16.01 & 0.7391 & 0.19 \\
1.5 & 0.095 & 0.8140 & 10.34 & 0.7391 & 0.11 \\
2.0 & 0.094 & 0.7906 & 7.17 & 0.7385 & 0.05 \\
2.5 & 0.092 & 0.7756 & 5.14 & 0.7381 & -0.92 \\
10 & 0.075 & 0.7220 & -2.13 & 0.7309 & \\
\hline
\end{tabular}


TABLe 3: Natural frequencies of the twofold solar array in the vertical direction.

\begin{tabular}{lcccc}
\hline $\begin{array}{l}\text { Suspension stiffness } k_{y} \\
\left(\mathrm{~N} \cdot \mathrm{mm}^{-1}\right)\end{array}$ & $\begin{array}{c}\text { Test value } \\
(\mathrm{Hz})\end{array}$ & $\begin{array}{c}\text { First-order natural frequency in the vertical direction } \\
\text { Relative error of test } \\
(\%)\end{array}$ & $\begin{array}{c}\text { Corrected value of mathematical model } \\
(\mathrm{Hz})\end{array}$ & $\begin{array}{c}\text { Corrected relative error } \\
(\%)\end{array}$ \\
\hline 0.0100 & 3.5211 & -2.03 & 3.6160 & 0.61 \\
0.0980 & 3.7884 & 5.40 & 3.5921 & 0.06 \\
0.1935 & 4.0536 & 12.78 & 3.5599 & -0.95 \\
0.2856 & 4.3000 & 19.64 & 3.5365 & -1.61 \\
0.3750 & 4.5300 & 26.04 & 3.5186 & -2.10 \\
0.4610 & 4.7475 & 32.09 & 3.5113 & -2.31 \\
\hline
\end{tabular}

is because that the test error is affected by different factors, and these factors are just approximately balanced. For example, the suspension stiffness and the pendulum effect make the test value larger than the true value, while the added mass makes it smaller than the true value. When the systematic errors caused by these two are equal, they offset each other. It makes the test error look small, but does not mean that the error does not exist or is proposed. When the test condition that makes error components offset each other cannot be predicted, error correction is still necessary.

Adopting the natural frequency identification method proposed in this paper, the relative error of the corrected first-order natural frequency in the horizontal direction is less than $1 \%$ and that in the vertical direction is less than $3 \%$. The identification accuracy of the ground modal test is improved by more than $50 \%$ after correction.

\subsection{Threefold Solar Array of Honeycomb Sandwich Plates.} There is a deployable solar array of threefolds applied to a small satellite of which the substrate is made of honeycomb Sandwich plates. For the plate, the single size is about $910 \mathrm{~mm} \times 1200 \mathrm{~mm}$ which weighs about $2.3 \mathrm{~kg} 2.6 \mathrm{~kg}$. The deployable size is about $2.92 \mathrm{~m}$. Each hinge weighs about $190 \mathrm{~g}$. Discretize parts of parameters and obtain the first four-order modes, as shown in Figure 9. In analysis, Mode 1 shows the first-order natural frequency in the horizontal direction, and Mode 4 shows the first-order natural frequency in the vertical direction.

The FEM model of the suspended modal test system is established for the threefold composite solar array, and test values of the first-order natural frequencies under different test conditions are obtained in the horizontal and the vertical directions. As above, the true natural frequencies are calculated with the encapsulated mathematical model and compared with the theoretical values, as shown in Tables 4 and 5 .

Using the proposed identification method, the relative error of the corrected first-order natural frequency in the horizontal direction is less than $0.5 \%$ and that in the vertical direction is less than $1 \%$. The identification accuracy of the ground modal test is improved by more than $50 \%$ after correction.

In conclusion, the natural frequency identification method of deployable solar arrays with multiple plates proposed in this paper can be applied to the ground modal test of solar arrays with current mainstream materials and configurations. With this method, the reliable identification of the first-order natural frequencies of solar arrays can be achieved through only one-time modal testing. However, the correction ability of modal test results differs for different types of solar arrays. In general, the proposed method has a slightly poor identification ability for the first-order natural frequency in the vertical direction of solar arrays with small size and small mass. The reason is that the mathematical model adopted in this paper considers each substructure as a particle, while the first-order natural frequency in the vertical direction will be affected by the mass distribution. Especially, the natural frequency of solar arrays with small size and small mass is more sensitive to the added mass, which results in a more serious error. Therefore, the added mass should be minimized when designing the test system. This is consistent with the design principle of modal tests.

In addition, the relative error of tests and the corrected relative error both reduce greatly when the rope length changes from $0.5 \mathrm{~m}$ to $1.0 \mathrm{~m}$ in Table 2 , and from $1 \mathrm{~m}$ to $2 \mathrm{~m}$ in Table 4. When it increases to $10 \mathrm{~m}$, the relative errors obviously reflect the negative impact of the added mass. Thus, when the rope length is about $2 \sim 5$ times of the plate length, there is a best-performance area for the testing error correction of the proposed identification method.

In theory, the smaller the suspension stiffness, the better the testing result. While it can be seen in Tables $2-5$, when the suspension stiffness is about $0.1 \mathrm{~N} \cdot \mathrm{mm}^{-1}$, the identification accuracy is better than $1 \%$ in all cases. The error correction effect is not effectively improved when the suspension stiffness even reducing to $0.01 \mathrm{~N} \cdot \mathrm{mm}^{-1}$. Considering the implementing difficulty of the test system, the suspension stiffness takes about $0.1 \mathrm{~N} \cdot \mathrm{mm}^{-1}$ that is enough.

\section{Test Verification}

The acrylic plate (a kind of transparent Plexiglas plate) is selected as the simulant of a solar array plate, and the rectangle-section aluminum beam is selected as a hinge simulant. This makes the structural composition of the simulant simple, and the dynamic characteristics easy to be determined. Therefore, the results of FEA under the ideal state can be taken as the theoretical frequency value of the test object. Based on FEA, the identification method proposed in this paper can be verified. Besides, the accuracy of the simulation and the identification method are discussed. The main material parameters have been measured by tests, as shown in Table 6. 


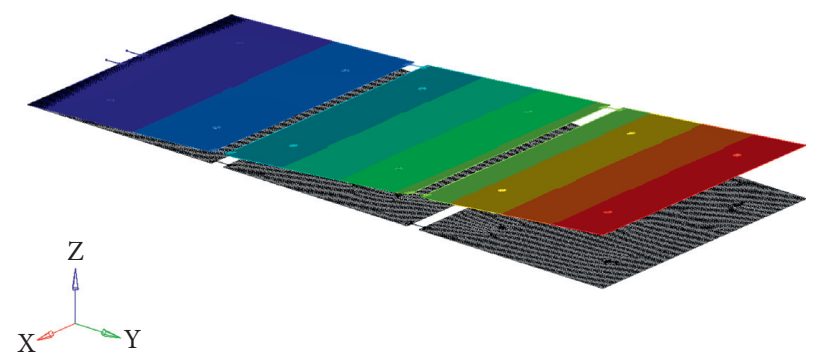

(a)

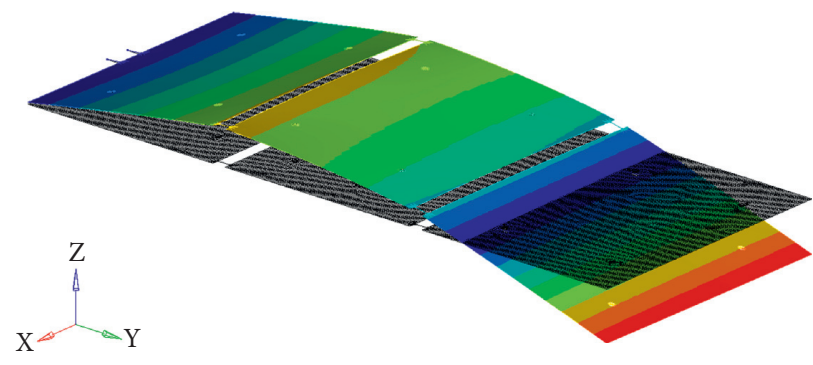

(c)

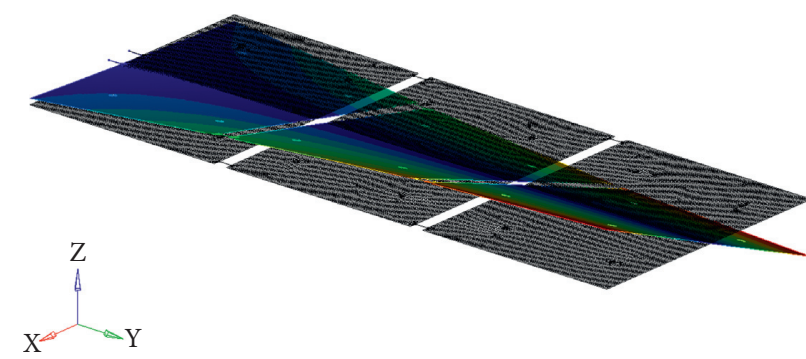

(b)

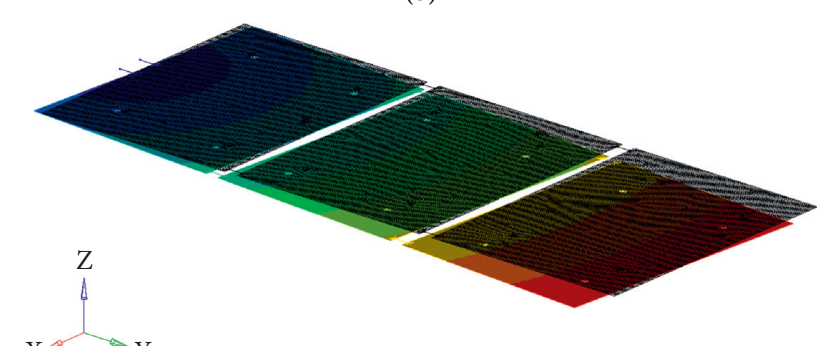

(d)

FiguRE 9: First four-order modes of the threefold solar array of honeycomb sandwich plates: (a) mode 1: freq. $=0.9 .556 \mathrm{~Hz}$; (b) mode 2: freq. $=2.6 .972 \mathrm{~Hz}$; (c) mode 3: freq. $=5.5 .747 \mathrm{~Hz}$; (d) mode 4: freq. $=5.8 .707 \mathrm{~Hz}$.

TABLe 4: Natural frequencies of the threefold solar array in the horizontal direction.

\begin{tabular}{|c|c|c|c|c|c|}
\hline \multirow[b]{2}{*}{ Rope length $L(\mathrm{~m})$} & \multirow[b]{2}{*}{ Suspension stiffness $k_{x}\left(\mathrm{~N} \cdot \mathrm{mm}^{-1}\right)$} & \multicolumn{4}{|c|}{ First-order natural frequency in the horizontal direction } \\
\hline & & $\begin{array}{c}\text { Test value } \\
(\mathrm{Hz})\end{array}$ & $\begin{array}{c}\text { Relative error of } \\
\text { test }(\%)\end{array}$ & $\begin{array}{l}\text { Corrected value of mathematical } \\
\text { model }(\mathrm{Hz})\end{array}$ & $\begin{array}{c}\text { Corrected relative } \\
\text { error }(\%)\end{array}$ \\
\hline 1 & 0.097 & 1.0198 & 6.72 & 0.9580 & 0.25 \\
\hline 2 & 0.094 & 0.9769 & 2.23 & 0.9571 & 0.16 \\
\hline 3 & 0.091 & 0.9589 & 0.35 & 0.9561 & 0.05 \\
\hline 4 & 0.088 & 0.9488 & -0.71 & 0.9551 & -0.05 \\
\hline 5 & 0.086 & 0.9423 & -1.39 & 0.9542 & -0.15 \\
\hline 10 & 0.075 & 0.9276 & -2.93 & 0.9514 & -0.44 \\
\hline
\end{tabular}

TABLE 5: Natural frequencies of the threefold solar array in the vertical direction.

\begin{tabular}{lcccc}
\hline $\begin{array}{l}\text { Suspension stiffness } k_{y} \\
\left(\mathrm{~N} \cdot \mathrm{mm}^{-1}\right)\end{array}$ & $\begin{array}{c}\text { Test value } \\
(\mathrm{Hz})\end{array}$ & $\begin{array}{c}\text { First-order natural frequency in the vertical direction } \\
\text { Relative error of test } \\
(\%)\end{array}$ & $\begin{array}{c}\text { Corrected value of mathematical model } \\
(\mathrm{Hz})\end{array}$ & $\begin{array}{c}\text { Corrected relative error } \\
(\%)\end{array}$ \\
\hline 0.0100 & 5.6059 & -4.51 & 5.8237 & -0.80 \\
0.0968 & 5.6861 & -3.14 & 5.8371 & -0.57 \\
0.1874 & 5.7660 & -1.78 & 5.8486 & -0.38 \\
0.2726 & 5.8380 & -0.56 & 5.8570 & -0.23 \\
0.3527 & 5.9027 & 0.55 & 5.8624 & -0.14 \\
0.4282 & 5.9606 & 1.53 & 5.8648 & -0.10 \\
\hline
\end{tabular}

TABle 6: Main material parameters of the ground verification test system.

\begin{tabular}{|c|c|c|c|c|c|c|c|}
\hline Material & & & & Main par & ters & & \\
\hline Acrylic plate & $\begin{array}{c}\mathrm{EX}(\mathrm{MPa}) \\
3522\end{array}$ & $\begin{array}{c}\mathrm{EY}(\mathrm{MPa}) \\
3730\end{array}$ & $\begin{array}{c}\text { GXY }(\mathrm{MPa}) \\
1282\end{array}$ & $\begin{array}{c}\text { GXZ (MPa) } \\
19300\end{array}$ & $\begin{array}{c}\text { GYZ }(\mathrm{MPa}) \\
19900\end{array}$ & $\begin{array}{c}\text { Poisson's ratio } \\
0.3\end{array}$ & $\begin{array}{c}\text { Density }\left(\mathrm{kg} \cdot \mathrm{t}^{-1}\right) \\
1.12 e-9\end{array}$ \\
\hline Aluminium alloy & & & $\begin{array}{c}E(\mathrm{MPa}) \\
68000\end{array}$ & & & $\begin{array}{c}\text { Poisson's ratio } \\
0.3\end{array}$ & $\begin{array}{c}\text { Density }\left(\mathrm{kg} \cdot \mathrm{t}^{-1}\right) \\
2.7 e-9\end{array}$ \\
\hline
\end{tabular}




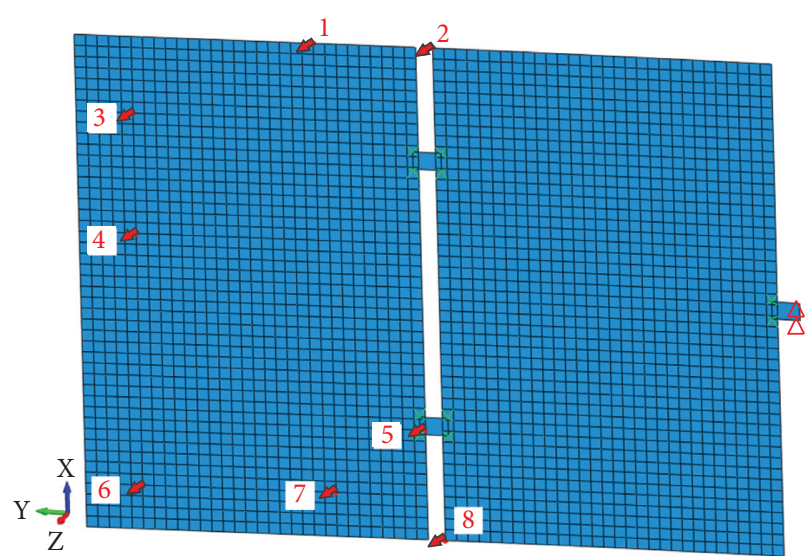

(a)

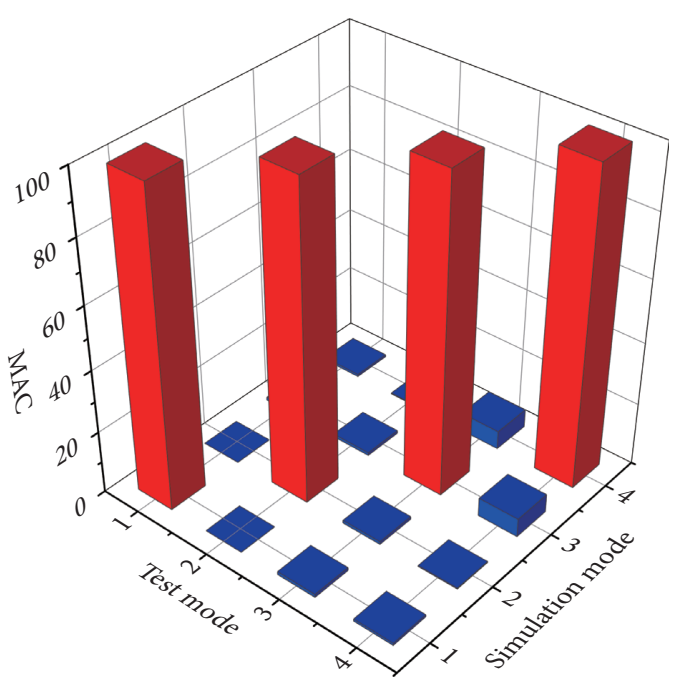

(b)

FIGURE 10: Optimal sensor placement: (a) sensor placement; (b) MAC matrix.

First, the sensor placement needs to be determined to ensure the effectiveness of modal test data. Based on the results of FEA, Mode 1 is the test object's first-order natural frequency in the horizontal direction, and Mode 4 is the test object's first-order natural frequency in the vertical direction. Therefore, the sensor placement is optimized for the first four-order modes. The results of the optimal sensor placement and the modal assurance criterion (MAC) Matrix are shown in Figure 10.

Before testing, mark the acrylic plates according to the optimal sensor placement and paste sensors. MSA1000A50 microelectromechanical systems (MEMS) accelerometers are selected to collect the simulant's acceleration data for modal parameter identification. The frequency response range of the accelerometer is $0 \sim 5000 \mathrm{~Hz}$, which can meet the test requirement of ultra-low frequency. Besides, the mass of a single sensor is only $5 \mathrm{~g}$, and it can be fixed by pasting, thus the added mass is small. Meanwhile, the stiffness of the suspension spring is $0.5 \mathrm{~N} \cdot \mathrm{mm}^{-1}$. The suspension rope, which is made of Kevlar, has a length of $4.3 \mathrm{~m}$ with a diameter of $1 \mathrm{~mm}$. And the rope's elastic modulus is about $3300 \mathrm{MPa}$. The DASP modal tester produced by China Orient Institute of Noise and Vibration is adopted. The test site is shown in Figure 11. As the acrylic board is made of transparent material, the adhesive tape is pasted around to show its outline. The intersections of dotted lines (including sides of the acrylic plates) indicate the position of the measuring points. The fixed end of the test object is connected to the fixed equipment. The fundamental frequency of the equipment is more than ten times of the measured frequency. The free end of the test object is suspended under the large truss by ropes. The rope length is exactly measured by the height adjusting module to make the indication of a single force sensor equal to the gravity of the corresponding substructure.

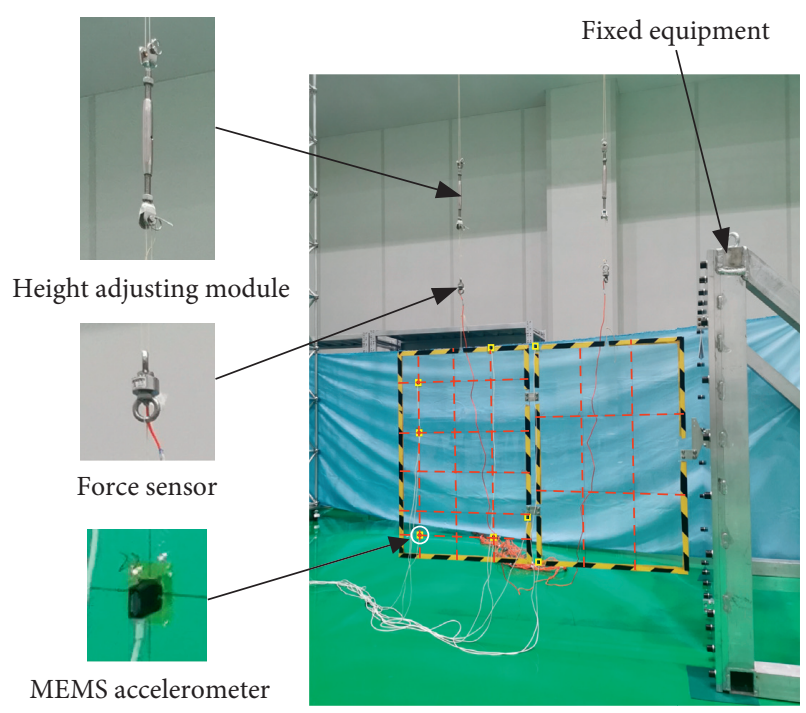

Figure 11: Schematic diagram of the test site.

The first five-order natural frequencies of the simulant are obtained through the ground modal test, as shown in Figure 12.

Establish the FEM model of the ground test system and obtain the first-order natural frequencies under testing of the solar array simulant, as shown in Table 7 . The result is very close to the test data, which prove that the simulation method is correct and the previous simulation data are reliable.

After the testing, the mathematical model matching with the test site is established. Test values and relevant test parameters are substituted into the mathematical model to obtain the equivalent parameters. Then, the true natural frequency of the solar array is identified using the test results, as shown in Table 8. 


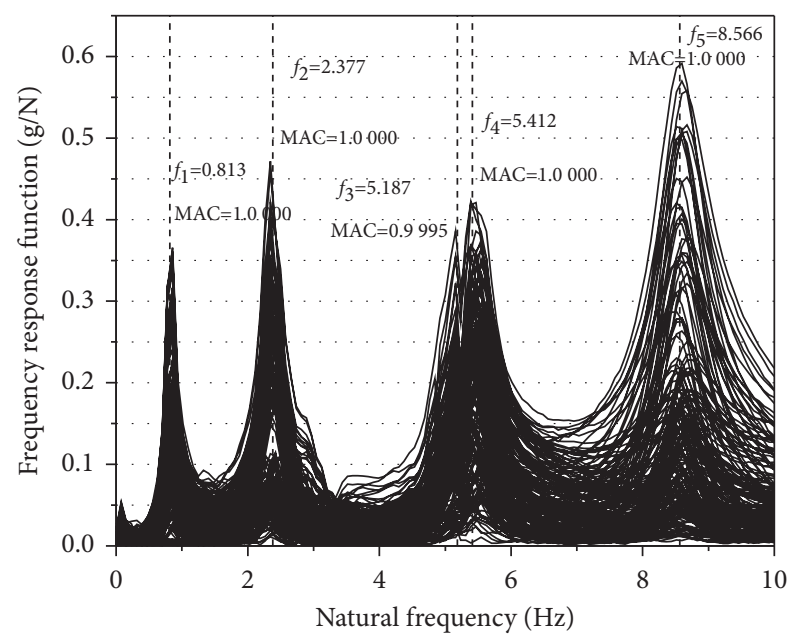

Figure 12: Frequency response function of the ground modal test.

TABLE 7: Simulation values and test values of the simulant.

\begin{tabular}{lcc}
\hline \multirow{2}{*}{ Condition } & \multicolumn{2}{c}{ First-order natural frequency } \\
& Horizontal direction & Vertical direction \\
\hline Test value $(\mathrm{Hz})$ & 0.813 & 5.412 \\
Simulation value $(\mathrm{Hz})$ & 0.812 & 5.435 \\
Relative error $(\%)$ & -0.12 & 0.42 \\
\hline
\end{tabular}

TABLE 8: Modal test results and corrected natural frequency of the simulant.

\begin{tabular}{lcc}
\hline \multirow{2}{*}{ Condition } & \multicolumn{2}{c}{ First-order natural frequency } \\
& Horizontal direction & $\begin{array}{c}\text { Vertical } \\
\text { direction }\end{array}$ \\
\hline Theoretical value $(\mathrm{Hz})$ & 0.807 & 5.474 \\
Test relative error $(\%)$ & 0.74 & -1.13 \\
Corrected value $(\mathrm{Hz})$ & 0.806 & 5.510 \\
Corrected relative error (\%) & -0.12 & -0.15 \\
\hline
\end{tabular}

It can be seen from Table 8 that the error between the corrected natural frequency and the theoretical value is less than $0.2 \%$. The identification accuracy is improved by more than $80 \%$. The results indicate that the suspension test system designed in this paper can achieve the effective modal testing of deployable solar arrays with multiple plates. Besides, the identification method proposed in this paper can identify the first-order natural frequencies of deployable solar arrays with multiple plates efficiently and reliably.

\section{Conclusion}

In this paper, a high-accuracy and high-efficiency method for natural frequency identification of deployable solar arrays with multiple plates is proposed. The proposed method does not need to establish a structural model with complete parameters but only needs parts of system parameters easy to be measured. Taking the mainstream solar array with multiple deployable plates as the research object, a suspended modal test system with gravity compensation function is designed according to the solar array's structure features. On this foundation, a mathematical model of the ground test system is established. To simplify the error correction process, the encapsulation method is adopted. Parts of the solar array parameters are abstracted as equivalent parameters. Using the encapsulation as a medium, the direct mapping relationship between the modal test result and the true natural frequency is established. By adopting the proposed method, less calculation is needed and the convenience for engineering application is increased. FEA and experimental verification show that the method proposed in this paper can be applied to the ground modal test of solar arrays with mainstream materials and configurations. Using simple and readily available system information, the natural frequency identification is achieved. The identification precision of the first-order natural frequency in the ground modal test increases up to $50 \%$. Meanwhile, the relative identification error of the first-order natural frequency after correction is within 3\%. Compared with the traditional ground identification methods, the proposed method omits complex processes of parametric testing and model updating. The requirements of accuracy and efficiency for the attitude control of spacecraft and the batch production of small satellites are met.

However, the proposed method has limitations. The theoretical model considers each substructure as a particle, which ignores the mass distribution. As the first-order natural frequency in the vertical direction will be affected by the mass distribution, the identification method is weak in correcting modal test results of small size solar arrays. Therefore, this method needs further study.

\section{Acronyms}

FEA: Finite element analysis

MIMO: Multi-input multi-output

FEM: Finite element method

DASP: Data acquisition and signal processing system

PCB: Printed circuit board

MAC: Modal assurance criterion

MEMS: Microelectromechanical systems.

\section{Data Availability}

The data used to support this study may be available from the corresponding author upon request.

\section{Conflicts of Interest}

The authors declare that there are no conflicts of interest regarding the publication of this paper.

\section{Acknowledgments}

Thank Changguang Satellite Technology Co., Ltd. for its experimental support and funding. Also, thank the satellite structure research laboratory for its technical support. This paper was funded by Changguang Satellite Technology Co., Ltd. and the Technology Development Program of Jilin Province (20210508038RQ). 


\section{References}

[1] M. K. Ben-Larbi, K. F. Pozo, M. Choi et al., "Towards the automated operations of large distributed satellite systems. part 1: review and paradigm shifts," Advances in Space Research, vol. 67, pp. 3598-3619, 2020.

[2] M. K. Ben-Larbi, K. F. Pozo, M. Choi et al., "Towards the automated operations of large distributed satellite systems. part 2: classifications and tool," Advances in Space Research, vol. 67 , pp. $3620-3637,2020$.

[3] D. Mascarenas, D. Macknelly, J. Mullins, H. Wiest, and G. Park, "Characterization of satellite components assembly for responsive space applications," Measurement Science and Technology, vol. 24, no. 7, Article ID 075101, 2013.

[4] J. Tao, T. Zhang, and Y. Nie, "Attitude maneuvering and vibration reducing control of flexible spacecraft subject to actuator saturation and misalignment," Shock and Vibration, vol. 2018, Article ID 3129834, 16 pages, 2018.

[5] Y. Xie, P. Liu, and G.-P. Cai, "On-orbit identification of flexible parameters of spacecraft," Proceedings of the Institution of Mechanical Engineers-Part K: Journal of Multi-Body Dynamics, vol. 230, no. 2, pp. 191-206, 2016.

[6] $\mathrm{S}$. $\mathrm{Li}$ and $\mathrm{Q}$. Wu, "Finite-time control algorithm based on modal state observer for flexible satellite attitude tracking," IEEE Access, vol. 8, pp. 184722-184730, 2020.

[7] K. Komatsut, M. A. Sanot, and A. Tsujihatai, "Experimental modal analysis for a satellite with flexible appendages," in Proceedings of the 17th International Modal Analysis Conference, Kissimmee, FL, USA, February 1999.

[8] S. J. Guo, H. Q. Li, and G. P. Cai, "Deployment dynamics of a large-scale flexible solar array system on the Ground," Journal of the Astronautical Sciences, vol. 66, no. 2, pp. 225-246, 2019.

[9] S. E. Woodard and J. M. Housner, "Nonlinear behavior of a passive zero-spring-rate suspension system," Journal of Guidance, Control, and Dynamics, vol. 14, no. 1, pp. 84-89, 1991.

[10] M. R. Ashory, "Correction of mass-loading effects of transducers and suspension effects in modal testing," Proceedings of SPIE-The International Society for Optical Engineering, vol. 2, 1998.

[11] R. Hunady and M. A. Hagara, "New procedure of modal parameter estimation for high-speed digital image correlation," Mechanical Systems and Signal Processing, vol. 93, pp. 66-79, 2017.

[12] D. Kumar, S. Kamle, P. M. Mohite, and G. M. Kamath, "A novel real-time DIC-FPGA-based measurement method for dynamic testing of light and flexible structures," Measurement Science and Technology, vol. 30, no. 4, Article ID 045903, 2019.

[13] Y. B. Zhang, W. J. Chen, X. W. Deng, Z. Y. Yu, C. Xie, and F. J. Peng, "Simulation and experiment for membrane modal identification in the air and low vacuum environment," Journal of Vibration and Shock, vol. 39, pp. 168-182, 2020.

[14] H. Luo, W. Wang, F. Jia, and L. Jiao, "Finite element model updating of satellite sailboard based on sensitivity analysis," Shock and Vibration, vol. 2019, Article ID 4547632, 12 pages, 2019.

[15] X. D. Li, W. Liu, and C. Z. Fan, "Dynamic characteristics of satellite solar arrays under the deployment shock in orbit," Shock and Vibration, vol. 2018, Article ID 6519748, 8 pages, 2018.

[16] V. Wickramasinghe, Y. Chen, D. Zimcik, P. Tremblay, H. Dahl, and I. Walkty, "Modal survey test and model correlation of the CASSIOPE spacecraft," Experimental Techniques, vol. 37, no. 6, pp. 15-23, 2013.
[17] H. Luo, W. Wang, J. Fu, and L. Jiao, "Finite element model updating of satellite sailboard based on sensitivity analysis," Shock and Vibration, vol. 2019, Article ID 4547632, 12 pages, 2019.

[18] Y. Xie, P. Liu, and G. Cai, "On-orbit frequency identification of spacecraft based on attitude maneuver data," Aircraft Engineering \& Aerospace Technology, vol. 90, no. 1, pp. 33-42, 2018.

[19] G. A. Tang, B. F. Chen, M. Y. Zhang, and Q. W. Guo, "Onorbit modal identification for vibration suppression of flexible aerospace structure using reaction wheel actuator," Aerospace Science and Technology, vol. 107, Article ID 106250, 2020.

[20] X. Zang, G. A. Tang, Q. W. Guo, and X. H. Dong, "Ground modal test and performance evaluation of large flexible accessories in spacecraft," Manned Spaceflight, vol. 23, pp. 556-563, 2017.

[21] S. Wu, Q. W. Guo, X. Zang, and T. Xu, "Error analysis for fundamental frequency measurement of a suspended modal test system," Journal of Vibration and Shock, vol. 37, no. 19, pp. 82-86, 2018. 